CHAPTER 8

\title{
COMPARATIVE MECHANISMS FOR TRANSCRIPTION AND REGULATORY SIGNALS IN ARCHAEA AND BACTERIA
}

\author{
AGUSTINO MARTÍNEZ-ANTONIO and JULIO COLLADO-VIDES
}

\section{Introduction}

The human genome and the microbial genomes represent two major frontiers in genomic studies. With the rapid advancement in genome sequencing techniques, the sequencing of a large number of bacterial and archaeal genomes has resulted in the largest increase in the number of new genes identified because of their diversified forms of life on the earth (Rusch et al., 2007; Yooseph et al., 2007). As we know, microbial systems span at least 3.8 billion years of evolution. They are persistent, ubiquitous, and represent the essential components of all ecosystems. The geo- and physicochemical composition of Earth's biosphere has been continuously molded by microbial activities. Until recently, there had been no agreement among investigators over the issue of diversity and fraction of the cultivated microorganisms and the way they evolve. The development of massive genome sequencing and molecular phylogenetic projects has recently enabled the characterization of naturally occurring microbial biota even without cultivation. Indeed, molecular sequences have enabled us to follow the way in which evolution works (Zuckerkandl and Pauling, 1965). Molecular structures and nucleic acid sequence analyses have suggested that life on this planet forms three distinctive groups of organisms: archaea, bacteria and eukarya (Woese et al., 1990). In general terms, archaea resemble bacteria as being single-cell organisms having similar cellular ultra-structure, genome size and organization. The evolution of archaea and bacteria is difficult to reconstruct, and it is speculated that the chronic energy stress might be the only driving-force for their cellular evolution (Valentine, 2007). The gene order maintained between archaea and bacteria depends to some extent on the conservation between their superoperonic elements encoded for RNA ribosomal proteins, proton-pump ATPase and ABC transport systems (Wolf et al., 2001). However, archaea manage their genetic information through a mixture of the molecular mechanisms occurring in eukarya and in bacteria (Bell and Jackson, 1998; Leigh, 1999; Bell and Jackson, 2001; Bell et al., 2001; Ouhammouch, 2004). The basal transcriptional machinery in archaea, that is the core promoter elements and the general factors for their recognition, is similar to that of eukarya, whereas 
gene organization and sequence-specific TFs (transcription factors) for regulating transcription are environmental and cellular condition-dependent as in bacteria. In this comparative study we describe and review our current knowledge about the transcriptional mechanisms and the regulatory signals involved in gene expression in the archaeal and bacterial domains. We focus on the distinct molecular mechanisms that ensure transcription: i) the basal machinery for the recognition of the core promoters including DNA sequences (cis-regulatory elements) and the general TFs (trans-regulatory factors) required for transcription initiation, and ii) the way gene transcription is modulated depending on nucleoid structure and through the activity of small ligands or environmental signals that specific TFs can bind or sense. Where possible, we describe in detail the biological processes from the beginning to the end of transcription as well as some additional mechanisms regulating gene expressions before mRNA translation.

\section{Gene Organization in Archaea and Bacteria}

The decision about which genes should be expressed at a specified time is very important because the cellular biochemical abilities and limitations are specified by the activities of the gene products. As transcription of genes is a very costly and time-consuming process, it is not surprising that the regulation of the expression of genes at the transcriptional level has been described as a tightly controlled event. Indeed, a recent analysis measuring mRNA and proteins content conclude that the cellular proteome profile could be defined as $\sim 70 \%$ and $\sim 50 \%$ solely by the transcriptional regulation in yeast and $E$. coli, respectively, while for the rest, the regulation occurs possibly at the translational and posttranslational levels. The weaker correlation observed in E. coli might be explained by the fact that in the bacterial operon structure, genes are co-transcribed but often differentially translated ( $\mathrm{Lu}$ et al., 2007).

\subsection{Operons and Transcription Units}

In prokaryotes (archaea and bacteria), most of genes tend not to be dispersed in genomes but frequently encoded by functional units of expression, firstly described as "operons" by Jacob and Monod (Jacob and Monod, 1961). They had initially defined an operon as a set of several genes that are transcribed in a single polycistronic unit (Fig. 1A). An operon has associated cis-regulatory elements: one or several operator sites (the DNA-sequence that is bound/recognized by specific TFs to modulate the promoter activity), a promoter (the region on DNA where the RNAP starts the transcription) upstream of all genes in the operon, and a terminator downstream (the region where transcription ends and RNAP dissociates from DNA). In all cases studied so far, one gene is associated with only one operon.

It is also relatively common to find operons with several promoters, some of them internally located, thus transcribing a partial group of genes in the operon. 


\section{A) Operon structure}

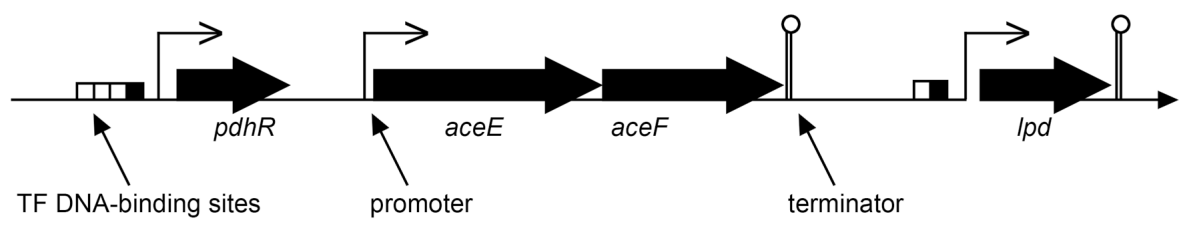

\section{B) Transcription Units}

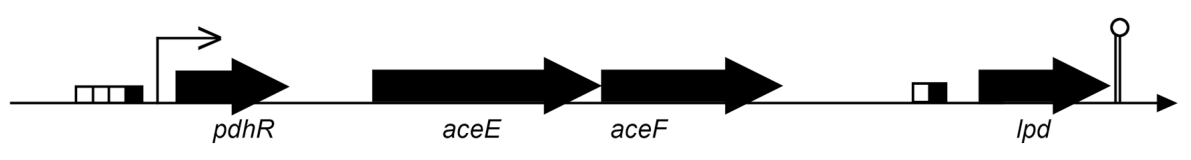

pdhR-aceEF-Ipd

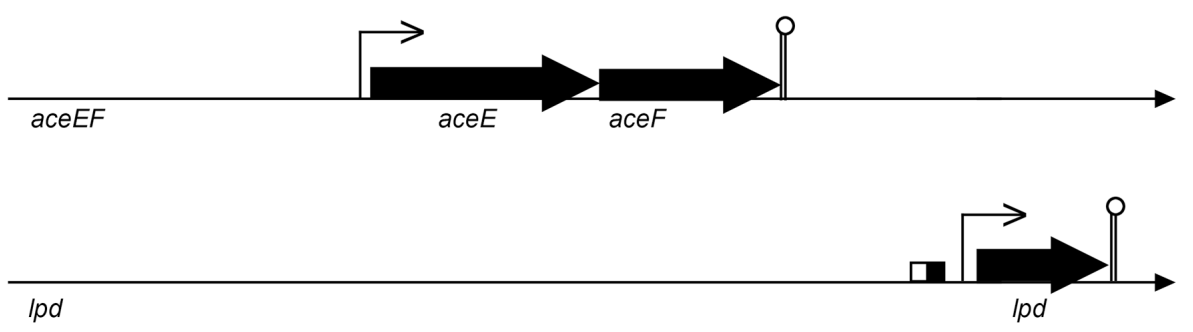

Fig. 1. Graphical representation of the genetic organization in bacteria and archaea. (A) The pdhR-aceEF-lpd operon of Escherichia coli K12 and their cis-regulatory elements, and (B) the transcription units included in this operon: $p d h \mathrm{R}-a c e \mathrm{EF}-l p d$, aceEF and $l p d$ encoding genes respectively (data from Regulon DB database, URL: http://regulondb.ccg.unam.mx/) (Salgado et al., 2006).

This partial group of genes constitutes a transcription unit (TU) that is a set of one or more genes transcribed from a single promoter. A TU may also include regulatory protein binding sites affecting the transcriptional activity of the promoter and a terminator signaling the end of transcription. A complex operon with several promoters contains, therefore, several TUs (Fig. 1B). At least one TU must include all the genes present in the operon, given the definition of an operon. Thus, a TU should be considered as the minimal unit of genetic information to be transcribed (as of one gene, Fig. 1B) and an operon is the maximal group of genes to be cotranscribed with the possibility to include more than one TU (Fig. 1A). Transcribed individual genes should be considered as TUs, in agreement with their original definition as they are not operons. Finally, a gene is the segment of DNA involved in producing a polypeptide chain or stable RNA; it includes regions preceding and following the coding region (leader and trailer), (Scherrer and Jost, 2007). More detailed discussions on operons are presented in Chapter 10. 


\section{Basal Factors and cis-Regulatory Elements for Core Promoter Recognition}

The metabolic, defensive, communicative and pathogenic capabilities of microorganisms depend primarily on the repertoire of genes encoded in their genome and their ability to regulate the expression of this information according to the changing environments. Thus, as a consequence of the endogenous and exogenous physicochemical setting, micro-organisms should turn on or off a specific set of genes to adapt to new milieu conditions. For the proper regulation of gene expression, the cellular machinery is required to read the genetic information encoded in the genome for which organisms use a set of Transcription Associated Proteins (TAP) including RNA polymerase (RNAP) constituents, basal or general factors for promoter recognition and specific TFs. Thus, the successful turn on or off of genes requires the transcriptional machinery must "interpret" correctly the "cisregulatory code" present at the beginning and terminus of the transcription units. In the following section, we describe the general or basal factors and the cisregulatory signals necessary for the proper recognition of promoter sequences for gene transcription initiation in archaea and bacteria, respectively.

\subsection{The Basal Transcription Machinery in Archaea}

In vitro experiments, mainly with hyperthermophilic archaea (the most genetically engineered archaeal model organism), show that the basal machinery for transcription in archaea is basically similar to that corresponding to RNAP II in eukaryotes (Bell et al., 1999, 2001; Hickey et al., 2002; Bartlett, 2005). In addition to the 12 subunits of RNAP, archaea need two general TFs: TBP and TF B, with the first one being homologous to the TATA-box binding protein (TBP) and the second one corresponding to TF IIB, both from eukaryotes, respectively (Fig. 2A). It has also been found that TF $\mathrm{E}$ is the third general TF in archaea, homologous to the minimal functional region of TF IIE from eukarya. Although it is not required for transcription in vitro, it can be used to validate the promoter recognition by TBP when it is sub-optimal. The function of the basal factors is to recognize and bind to specific operator DNA sequence near promoters in the transcription units, and to recruit the RNAP to make up the protein complex essential for initiating the transcription in a unidirectional way.

The DNA elements in the regulatory region needed for transcription include: i) the TATA-box with an AT-rich DNA sequence of $8 \mathrm{bp}$ in length (formerly called box A) localized around $24 \mathrm{bp}$ upstream of the transcription initiation site. This TATA-box site is recognized and contacted by the TBP (TATA binding protein) factor; and ii) the BRE element (for TF B-responsive element), an additional purine-rich element localized immediately upstream of the TATA-box. This site is important for maintaining the promoter strength, since it is recognized and contacted by the carboxy-terminal domain of TF B. BRE is the principal element 
A)



B)
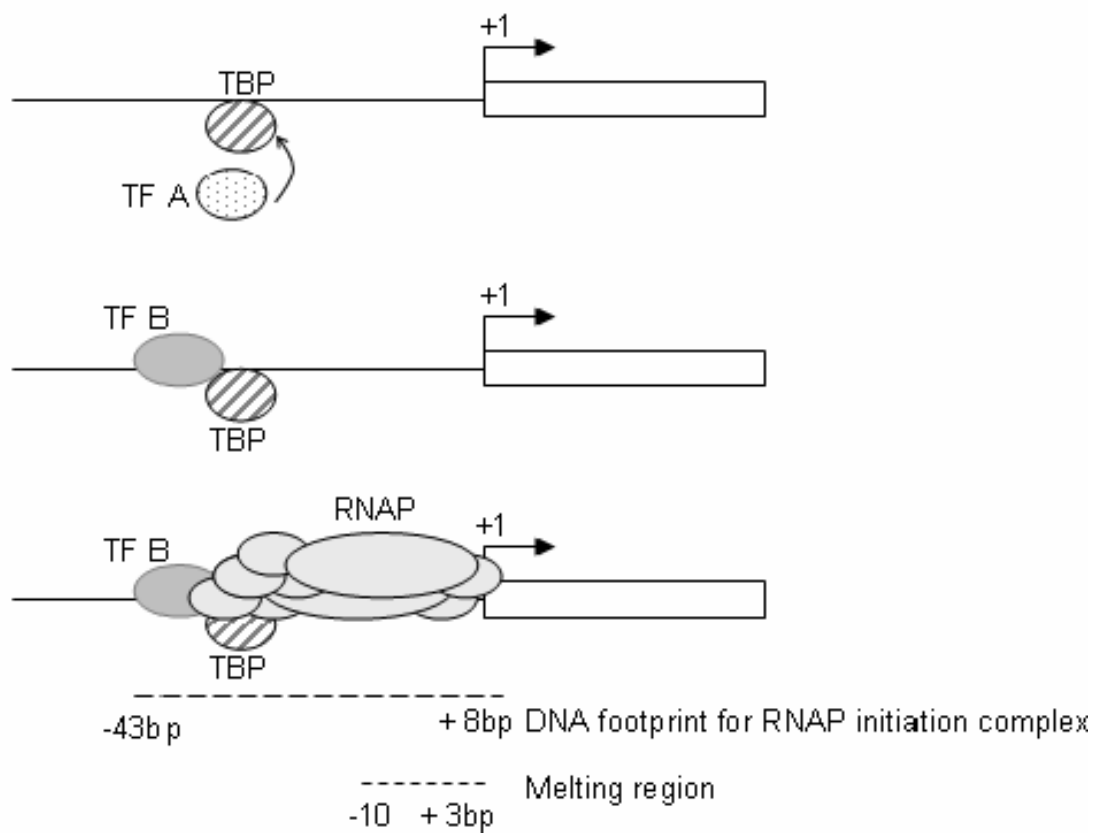

Fig. 2. Basal transcription machinery in archaea. (A) cis-regulatory elements for basal promoter recognition. (B) Recruitment of general trans-regulatory factors to initiate transcription. BRE, TF B-responsive element; TBP, TATA-box binding protein; TF E, transcription factor E; TF B, transcription factor $\mathrm{B}$.

for governing the oriented assembly of the transcription pre-initiation complex since TF B with their N-terminus recruits the RNAP and ensures the proper orientation on the promoter for initiating transcription.

The molecular events responsible for the transcription have not been understood totally. The following sequential events are postulated:

1. TBP is bound to the TATA-box, sometimes assisted by the TF E factor.

2. Subsequently, TF B is bound to the TBP-DNA complex by making contact with the BRE site.

3. Then, the N-terminus of TF B recruits RNAP and orients it properly to form the pre-initiation complex. The corresponding DNase footprint extends from -43 to at least +8 bp with respect to the transcription initiation site.

4. In this pre-initiation complex, RNAP catalyzes the isomerization of the promoter DNA by melting a region of about 13 bp extending from around -10 to $+3 \mathrm{bp}$. 
5. Subsequently, RNAP initiates RNA synthesis resulting in a RNAP-DNA-RNA complex around the first 5-6 nucleotides of the transcript.

6. Finally, the transition between a promoter-bound to an elongated form of the RNAP clears the promoter. The process of RNAP escape from the promoter seems to be facilitated by an N-terminal sequence, which is conserved between the zinc ribbon and the core domain of the same TF B (Fig. 2B).

It is particularly interesting that some archaea encode multiple paralogs of TF $\mathrm{B}$ and/or TBP. This finding raises the possibility of differential usages of these basal factors, which entails a general mechanism for regulating gene transcription similar to the multiple $\sigma$ factors present in bacteria (see below). This should happen when individual paralogs of $\mathrm{TF} B$ recognize different BRE sequences, allowing for discrimination between different promoter subtypes. Consistent with this hypothesis, it has been found that one TF B is expressed following a heat shock exposition in Haloferax volcanii, which might indicate a condition-specific expression and activity of this TF B (Facciotti et al., 2007).

\subsection{The Basal Transcription Machinery in Bacteria}

The basal molecular machinery for transcription in bacteria is different from that present in archaea and eukarya. The main component responsible for transcription in bacteria is the multi-subunit RNAP, but not in a promoter-specific manner as it mediates but not initiates the process (Collado-Vides et al., 1991; Browning and Busby, 2004). The RNAP core consists of only 5 subunits: $\alpha \mathrm{I}, \alpha \mathrm{II}, \beta, \beta^{\prime}$ and $\omega$ with a combined molecular mass of $\sim 400 \mathrm{kDa}$. The $\beta$ and $\beta^{\prime}$ subunits $(1,324$ and 1,047 residues, respectively, in Escherichia coli) constitute the largest RNAP component and contain the active site required for the binding of both template of DNA and the RNA product during transcription. The two $\alpha$ subunits (each 329 residues) each consist of two independently folded domains joined by a $\sim 20$ amino-acids flexible linker. The larger N-terminal domain $(\alpha$-NTD) dimerizes and is responsible for the assembly of $\beta$ and $\beta^{\prime}$ subunits. The smaller carboxyl-terminal domain ( $\alpha$-CTD) is a DNA-binding domain with an important role in certain promoters presenting $U P$ elements (see Sec. 3.4). Finally, the small $\omega$ subunit (91 residues) seems to play a chaperone role in the assembly of the $\beta^{\prime}$ subunit. In order to regulate transcription by a particular promoter, the core RNAP must first interact with a particular $\sigma$ factor to form the RNAP holoenzyme (Fig. 3). In this way, the promoter sequences correspond to a variety of $\sigma$ factors associated to the RNA polymerase core (Stragier and Losick, 1990; Gourse et al., 2000; Wade et al., 2006). The $\sigma$ factor has three main functions: (i) to recognize specific promoter sequences, (ii) to position the RNAP holoenzyme to specific target promoters, and (iii) to facilitate unwinding of the DNA duplex near the transcription initiation site. 


\subsection{The $\sigma^{70}$ and $\sigma^{54}$ Factor Families in Bacteria}

Most free-living bacteria contain multiple $\sigma$ factors to help them to adapt to changing environmental conditions. These $\sigma$ factors range from one $\sigma$ factor in Mycoplasma genitalia to seven in Escherichia coli and around 60 in Streptomyces (Ishihama, 2000; Gruber and Gross, 2003; Paget and Helmann, 2003). Each $\sigma$ factor preferentially recognizes variations in the promoter sequence, resulting in the regulation of the primary mechanism of differential gene transcription. Except for the $\sigma^{54}$ family, $\sigma$ factors belong to a large phylogenetic group namely the $\sigma^{70}$ family. Members of this family are the dominant $\sigma$ factors for promoter recognition in bacteria (the housekeeping $\sigma$ factor). $\sigma$ factors are multi-domain proteins with domains 2, 3 and 4 known to be involved in the promoter recognition; domain 1 is actually absent from many $\sigma$ factors. The $\sigma^{70}$ family can in turn be further classified into four groups: the housekeeping sigmas $(\sigma)$, sigmas that respond to general stress conditions, sigmas responsible for heat shock, motility and sporulation, and sigmas known as extracytoplasmic factors (ECF) originally identified as $\sigma$ factors associated with extracellular or membrane functions, and frequently co-transcribed with anti- $\sigma$ regulatory factors.

\subsection{Cis-Elements in $\sigma^{70}$-Depending Promoters}

Four different DNA-sequence elements for basal transcription have been identified in bacteria, with the first two being the more important ones: (i) one hexamer localized at -10 bp corresponding to the transcription initiation (the PribnowSchaller box). This -10 element resembles the TATA-box site and is recognized by subunit 2 of the $\sigma$ factor of the RNAP holoenzyme; (ii) the -35 bp promoter element to be recognized by the domain 4 of a $\sigma$ factor. The two other promoter signals include a $-10 \mathrm{bp}$ extended element and the $U P$ element. The extended -10 element is a 3-4 bp sequence located immediately upstream of the -10 hexamer and is recognized by domain 3 of a $\sigma$ factor. The $U P$ element is a $\sim 20$ bp sequence located upstream of -35 hexamer that is recognized by the $\mathrm{C}$ - terminal domains of the RNAP $\alpha$ subunits. The UP element, initially found in a few strong promoters and shown to be the point of contact for the RNAP $\alpha \mathrm{CTD}$, may well be present in a larger number of promoters (Haugen et al., 2006), (Fig. 3A).

In bacteria, the combination of $-10,-35$, extended -10 and $U P$ elements provides the basis for formation of a great diversity of specific sequences for the initial binding of RNAP. Like in archaea, the role of these elements in bacteria is to recruit the RNAP machinery to form an open-complex to be structurally ready for binding for initiating transcription. In general, any deficiency in one of these elements can be compensated by the other (Gruber et al., 2001; Mooney and Landick, 2003; Miroslavova and Busby, 2006; Miroslavova et al., 2006). The transcription process involves three main steps: initiation, elongation, and termination. 


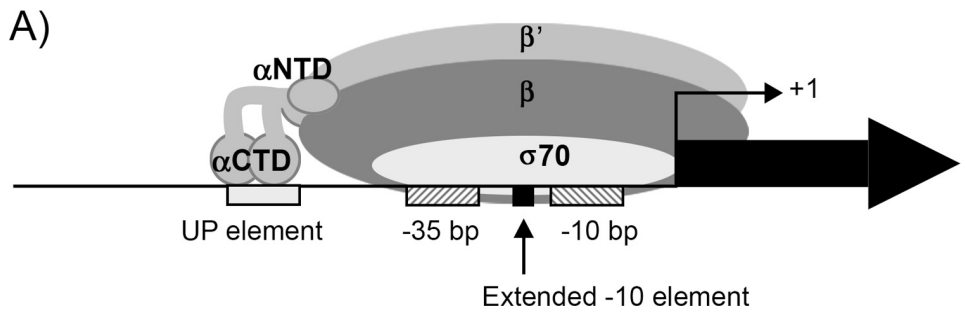

B)

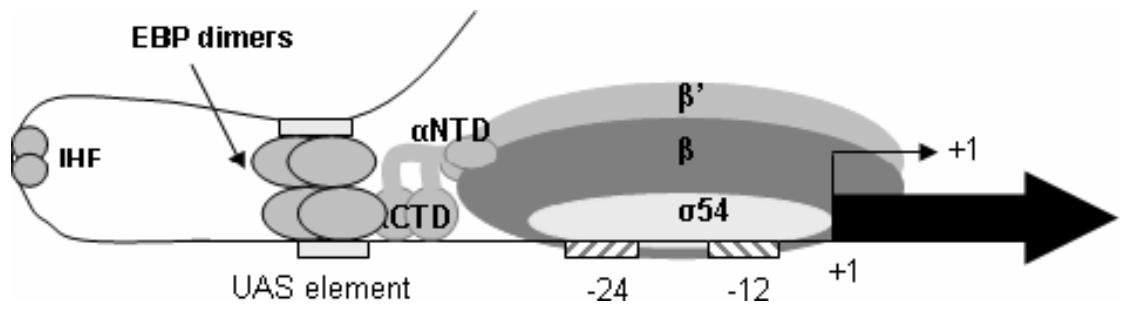

Fig. 3. cis-elements and trans-regulatory factors for basal transcriptional machinery in bacteria (A) in $\sigma^{70}$ promoters, and (B) in $\sigma^{54}$ promoters. The mode of activation at a distance in $\sigma^{54}$ promoters is illustrated. Note that the AAA + domain-containing activators can interact with $\sigma^{54}$ directly.

1. Transcription begins with the binding of the RNAP holoenzyme to the $-10,-35,-10$ extended and $U P$ elements responsible for the initiation of transcription to form the close-complex promoter.

2. After the initial binding of RNAP to the promoter region, the DNA sequence from around -10 to +2 bp downstream of the transcription activation site is unwound to form the open-complex bubble.

3. This strand isomerization allows the movement of the free DNA-template to the active site of RNAP to initiate the synthesis of RNA in the presence of nucleotides triphosphates (NTPs).

4. After the synthesis of $\sim 9-12$ nucleotides of RNA, the RNAP escapes from the promoter region followed by the beginning of the elongation stage.

5. At this point the RNAP undergoes a conformational change, leading to dissociation of RNAP-DNA contacts with the $\sigma$ factor.

\subsection{Bacterial $\sigma^{54}$-Dependent Promoters}

The family of $\sigma^{54}$ factors differ in their amino acid sequence and transcription mechanism from the $\sigma^{70}$ family (Xu and Hoover, 2001; Cases et al., 2003b). In spite of the lack of significant sequence similarity between these two groups of $\sigma$ factors, they bind to the core RNAP, although they produce holoenzymes with different mechanisms. The mechanism of action associated with the $\sigma^{54}$ factor involves distant transcriptional activation (earlier considered to be unique for eukarya organisms) by Enhancer Binding Proteins (EBP; see below). Recently, these 
TFs have been classified into the AAA+ protein superfamily (ATPases associated with various cellular activities) (Burrows, 2003; Lilja et al., 2004; Sallai and Tucker, 2005; De Carlo et al., 2006; Dago et al., 2007). Bacterial $\sigma^{54}$-RNAP is the target for sophisticated signal transduction pathways involving activation through remote enhancer cis-elements localized more commonly in the upstream promoter regions. Activation of $\sigma^{54}$-RNAP promoters requires specialized transcription factors EBP that recognize the enhancer-binding sites as well as the regulation of ATP or GTP hydrolysis to trigger the transcription. Contrary to $\sigma^{70}$, the $\sigma^{54}$ factor can recognize and bind their promoters in the absence of the core polymerase. The cis-regulatory elements necessary for the $\sigma$ factor recognition on $\sigma^{54}$ promoters include short DNA segments at -12 and -24 bp from the onset of transcription. Mutational analyses suggest that the -24 element contributes more to the promoter recognition compared to other elements. The transcription initiation rates on these promoters are controlled via regulation of the DNA melting step (open-complex promoter). The $\sigma^{54}$ holoenzyme forms a closed-complex and occupies the promoter in this state prior to activation; this closed-complex is unusually stable, and it does not spontaneously isomerize into an open-complex. In this way, transcription initiated by the $\sigma^{54}$ promoters is tightly controlled with a low level of escape. Therefore, these kinds of promoters are not subjected to repression, and their transcriptional activity can be controlled at varying levels depending on the presence of cognate activator protein (EBP) and the environmental stimuli. Thus, at $\sigma^{70}$ promoters, the TFs recruit RNAP to DNA, whereas at $\sigma^{54}$ promoters, the TFs work on a stably bound RNAP.

Different than $\sigma^{70}$ factors that are present in many copies of orthologous genes per bacterial genome, the $\sigma^{54}$ factor, commonly found as a single copy, is absent in some bacteria with reduced genomes (e.g., intracellular parasites), and is rarely found with two copies such as in Bradyrhizobium, Rhodobacter and Rhizobium. In principle, the main disadvantage of $\sigma^{54}$ promoters is the necessity of long stretches of intergenic DNA for looping mechanism of activation, and thus the necessity for larger chromosomes. However, a prevalent organization involves IHF binding sites, which bind the DNA, developing a specific physical proximity between the bound RNAP and the upstream activation factor. This oriented binding can, in principle, provide the basis for the specificity of activation of regulators by specific promoters, even in small chromosomes as those of bacteria (Gralla and Collado-Vides, 1996) (see the following section on nucleoid-associated proteins). Indeed, this is true for the proper regulation of completely isolated $\sigma^{54}$ promoters to inhibit cross-talks. Thus, evolutive mechanisms working for chromosome compactness might in turn inhibit the activity of $\sigma^{54}$ promoters in bacteria. Their kinetic properties make $\sigma^{54}$ regulons and stimulons to be the equivalent to closed-complexes that are the sets of genes whose expression might have been fully absent for a long time without having even any effect. It is only when the corresponding activator(s) appear that their expression initiates, as opposed to the $\sigma^{70}$ transcription that is leaky and therefore constant repression would be needed to have it silent for a long time. 


\section{Transcription Termination}

RNAP responds to two distinct sets of mRNA-sequence signals for transcription termination: i) the Rho-dependent terminators that require the Rho protein as an auxiliary factor; and ii) the Rho-independent terminators, or intrinsic terminators that are functional in the absence of additional factors (Abe et al., 1999; de Hoon et al., 2005; Mooney et al., 2005; Adelman et al., 2006; Banerjee et al., 2006; Ciampi, 2006; Kingsford et al., 2007).

\subsection{Rho-Dependent Transcription Termination in Bacteria}

Rho-mediated transcription termination requires both cis-regulatory elements and trans-regulatory factors on the mRNA: the only cis-regulatory elements common to all Rho-dependent terminators are generally unstructured, C-rich and G-poor, with little sequence conservation. The trans-regulatory factor Rho protein, encoded by the rho gene, is a homohexamer protein universally distributed and essential to bacteria. Most of the mechanism for the Rho activity is unsolved and the initial evidence suggests that Rho is a RNA/DNA helicase or translocase that binds to untranslated naked RNAs and terminates the mRNA synthesis by dissociating RNA polymerase from the DNA template (to release mRNA). The energy used in the process is derived by hydrolyzing ATP through its RNA-dependent ATPase activity. It has been proposed that Rho acts on RNAP via an interaction with the NusG protein; NusG is an essential bacterial protein modulator of the transcriptional elongation and termination events, and interacts directly with RNAP and the Rho protein (Banerjee et al., 2006; Ciampi, 2006; Landick, 2006).

\subsection{Rho-Independent Transcription Termination in Bacteria}

On the other hand, the intrinsic terminators (Rho-independent) contain a short $\mathrm{G}$ :C-rich palindromic sequence followed by a run of 6-8 U (uraciles) residues. The $\mathrm{G}$ :C rich sequence triggers the formation of stem-loop secondary structures in the mRNA upstream sequence leading to termination near the end of the run of Us. These stem-loop structures motivate pausing of the RNA polymerase, and the terminator has been proposed to destabilize the interaction between the paused RNAP and the template DNA. This destabilization could occur by a decrease in the stability of the RNA:DNA hybridization in the transcription bubble, by direct interactions between the RNA hybrid and the RNA polymerase, or by a combination of these effects (Abe et al., 1999; Yachie et al., 2006).

\subsection{Transcription Termination in Archaea}

Knowledge about transcription termination in archaeal genomes is rather limited. Evidence obtained from experiments on Methanothermobacter thermautotrophicus 
suggests that RNAP is subject to intrinsic termination signaled by an intergenic sequence. These preliminary results suggest that transcription can be triggered by an upstream sequence which provokes response to termination of the archaeal RNAP at a remote downstream sequence. However, confirmation of these hypotheses still needs validation.

On the other hand, the Rho protein seems to be absent in archaeal genomes. A bacterial-like NusG protein has been suggested to be present in archaea based on sequence similarity with the Thermus thermophilus NusG. In addition, this protein shares stretches of sequence similarity to the eukaryotic transcription elongation factor Spt5. The analysis of the three-dimensional structure of this factor suggests that although there is a clear evolutionary and functional relationship between the archaeal and bacterial NusG proteins, the structural, sequential and biochemical data reveal that many differences exist in their binding specificities to both nucleic acids and other proteins (Reay et al., 2004; Santangelo and Reeve, 2006). Therefore, the final functional evidence is required to prove the occurrence of Rho-dependent termination in archaea as well.

\section{Transcriptional Regulation by TFs}

The way by which bacteria and archaea couple the transcription of genes with the external and internal conditions is the use of regulatory proteins, specifically the TFs (Ptashne and Gaan, 2002; Adhya, 2003; Cases et al., 2003a; Barnard et al., 2004; Miroslavova and Busby, 2006). A TF is a protein (more precisely a protein complex, since it can be a dimer or multimer) that activates or represses the expression of transcription units upon binding to specific DNA-binding sites (cisregulatory elements). Historically, binding sites for transcriptional regulators were called operator sites. Operator sites in their more general definition are sites on the genomic DNA for the binding of repressors and activators. In bacteria, specifically for $\sigma^{54}$ promoters, the term "UAS" used for upstream activator sites also refers to activator sites that function remotely. A related term is enhancers, more commonly used for activators in eukarya. An enhancer has been initially defined as an activator site for EBPs that function from far upstream, in either orientation in relation to the promoter.

\subsection{Transcriptional Signal Sensing}

TFs have been considered for a long time as "two-headed" molecules (Jacob, 1970) with one domain for DNA sequence recognition - formally considered equivalent to the enzymatic site - and the other domain for the binding/sensing of small molecule effectors. In this way TFs could be considered as molecular switches that have an input module for signal sensing and an output module for the transcriptional response (Wall et al., 2004; Seshasayee et al., 2006). In bacteria, most TFs have these "two-headed" molecules contained in one single protein except 
in the signal transduction two-components systems where these modules belong to two different proteins; a protein containing the sensor component mostly localized in the periplasm for sensing exogenous signals, and a response regulator localized in the cytoplasm (Aravind et al., 2005; Ulrich et al., 2005; Alm et al., 2006). As a consequence, the mechanism of signal-dependent regulation is different in these two groups of TFs; in the first group the TF is regulated by allosterism through the binding of specific signals whereas in the second group a phosphorylation cascade from the sensor to the response component takes place to modify the TF covalently.

\subsection{Exogenous and Endogenous Signal Sensing by TFs}

All organisms sense and respond to environmental conditions and most of their physiology is remodeled according to changes in the milieu (Martinez-Antonio et al., 2003; Balazsi et al., 2005). Consequently, most cellular modifications occur in response to changes in the exterior. At least two main kinds of exogenous signals could be distinguished; those physicochemical signals that stress or damage the cell (e.g., osmotic pressure, high/low temperature, high/low pH, etc.) and those components introduced into the cell and further metabolized for fuel or use as building blocks. For sensing the first kind of extracellular signal, unicellular organisms mostly use the sensor proteins of the two-component systems; these proteins sense exogenous signals or their effect on the membrane structure (damage) and communicate this condition to the corresponding pair of response regulators in the cytoplasm through a phosphorylation cascade to generate a transcriptional/cellular response to alleviate this stressing condition. In the second group, the cell uses mostly transport systems inserted in the membrane that sense and import exogenous compounds. Both these transcriptional sensing mechanisms are used to monitor the exogenous conditions; the genetic elements involved (sensor/response (TF) components and $\mathrm{TF} /$ transport systems) tend to be encoded together in the E. coli chromosome (Martinez-Antonio et al., 2006; Janga et al., $2007 \mathrm{~b})$.

Imported metals, metabolites and some small diffusible components are further utilized by the cellular metabolism (catabolism and biosynthesis) required for cell functions. During these cellular processes, different metabolites are produced as intermediates or waste products. The concentration of some intermediate metabolites or potentially dangerous waste products merit cellular monitoring. Certainly, there is a set of TFs for sensing/binding these key metabolites (e.g., pyruvate, tryptophan, ATP levels, etc.) and dangerous conditions (e.g., redox potential, DNA damage, acetate accumulation, etc.), which are considered as TFs sensing endogenous conditions. Because the metabolism depends on external conditions or compounds, it is not surprising that the transcriptional regulation of both processes is coordinated (Balaji et al., 2007; Janga et al., 2007a). Thus, the TFs responsible for sensing the internal conditions are frequently regulating those TFs governing the functioning of transport systems in E. coli (Martinez-Antonio 
et al., 2006). Thus, TFs for internal sensing are those whose allosteric metabolite or equivalent signal is generated inside the cell. Curiously, internal sensing TFs are mostly global regulators that affect the transcription of a large number of genes, including other TFs of external sensing as well as those of dual sensing whose signal may be either synthesized internally or coming from the outside. Thus, global regulators in bacteria -at least those of the E. coli regulatory network-modulate a large number of genes in response to changes in the internal milieu of the cell. They alter a large number of genes' functional states directly through affecting their transcription, as well as regulating the expression of alternative TFs and additional sigma $(\sigma)$ factors (Martinez-Antonio and Collado-Vides, 2003).

\subsection{Additional Mechanisms Regulating the TF Activity}

In addition to the regulation of TF activity by effector signals or metabolites, there are other less known mechanisms for controlling the TF transcriptional activities:

(i) Cellular sequestration is exemplified by MalT (a regulator for maltose degradation in E. coli); in the absence of the transportable sugar, some components of the transport system (MalK, MalY) sequester the MalT regulator in the cellular periplasm, preventing the transcription of more transportable components until cell faces deficiency in terms of favorable/degradable sugars in the milieu and maltose is present for transportation and degradation (Schlegel et al., 2002).

(ii) A second mechanism is allowed by spatial/temporal availability of TFs as it happens in unequally dividing cells in $\alpha$-proteobacteria. One well studied model is Caulobacter crescentus where a master response regulator, CtrA, directly controls the initiation of chromosome replication as well as several aspects of polar morphogenesis and cell division. The CtrA activity is temporally and spatially regulated by multiple partially redundant control mechanisms, such as transcription, phosphorylation, and targeted proteolysis (McAdams and Shapiro, 2003; Ryan and Shapiro, 2003).

(iii) Another mechanism (related to those described in point i) is by activity, depending on the sub-cellular location of the regulator, as in the case of PutA (Proline utilization A), a multifunctional protein that in solution represses transcription of genes for proline utilization, whereas it is mobilized to the membrane when it is bound to proline to catalyze the oxidation of proline to glutamate (Zhu et al., 2002; Zhu and Becker, 2003). A similar case is that of the carbon source Mlc transcriptional regulator (Seitz et al., 2003). In eukaryotes, where TFs must enter the nuclei to be effective, sub-cellular location is believed to play a more important role (Holstege et al., 1998; Jans and Hassan, 1998).

(iv) In yet another interesting mechanism, the cellular concentration of a TF entails a balance between protein expression and degradation as is apparently 
the case for MarA and SoxS regulators in E. coli. In fact, in many cases the precise mechanism is unknown that degrades and reduces the concentration of the active TFs once their intended goal is accomplished. A possible mechanism is simple dilution by cell duplication, as has been demonstrated in the case of the LacI regulator (Elf et al., 2007). MarA and SoxS appear to interact with RNAP in solution, in the absence of promoters, "capturing" RNAP for use at certain transcription units. It is worth noting that this mechanism is similar to the one used by alternative $\sigma$ factors (Browning and Busby, 2004).

\section{Mechanisms of Gene Regulation by TFs}

\subsection{Transcriptional Regulation by Activators in $\sigma^{70}$ Promoters}

Activation is required when promoter recognition by RNAP is inefficient and an activator compensates for and attracts RNAP to the promoter. In general, activators bind target DNA sites located near the upstream regions on promoters (Gralla and Collado-Vides, 1996; Barnard et al., 2004). The position of these DNA binding regions could range from those located between the -35 and $-10 \mathrm{bp}$ elements to allow realignment of these cis-elements and their proper recognition by RNAP holoenzyme (Gralla, 1996; Rhodius and Busby, 1998; Lloyd et al., 2001). They also occur at regions upstream to the $-35 \mathrm{bp}$ sites that permit control of the TF with the $\alpha \mathrm{CTD}$ of RNAP to position it at the promoter. A related mechanism of activation, by recruitment, involves targeting a region adjacent to the promoter -35 element (the bound activator interacts with domain 4 of $\sigma^{70}$ ) by TF.

\subsection{Transcriptional Regulation by Repressors in $\sigma^{70}$ Promoters}

The role of repressor proteins is to reduce the transcription initiation through the most common and simple mechanism of binding to a DNA region that interferes with the promoter recognition by the RNAP. Another mechanism is, through polymerization, binding of a TF repressor to distant regions of the promoter but inducing DNA looping to make the promoter recognition by the RNAP inefficient, such as with GalR and DeoR TFs. The third mechanism described is the antiactivation in which a repressor binds to an activator affecting its contacting RNAP, particularly within the $\alpha$ CTD region (Rojo, 2001; Browning and Busby, 2004).

\subsection{Transcriptional Activation in Bacterial $\sigma^{54}$ Promoters}

The mechanisms described above apply to $\sigma^{70}$-dependent promoters, which are the most common promoters in the bacterial cell. The mechanisms available for regulation of $\sigma^{54}$ promoters are rather different (Kustu et al., 1989; Studholme and Dixon, 2003; Rappas et al., 2007), since, as described earlier, the RNAP coupled to a $\sigma^{54}$ factor requires EBPs to form the open-complex promoter to initiate 
transcription. As opposed to $\sigma^{70}$ promoters, there is no basal transcription of $\sigma^{54}$ promoters in the absence of activation. These EBPs could bind cooperatively as dimers to DNA sequences between 70 to $150 \mathrm{bp}$ upstream of the $\sigma^{54}$ promoters, but they can be active as far as $3 \mathrm{~kb}$ upstream. Oligomerization of the EBP induces a DNA loop, which permits the EBP to contact the RNAP $\sigma^{54}$-holoenzyme (Fig. 3B). The DNA loop could be randomly and transiently formed, but its intrinsic curvatures or the presence of some nucleoid associated proteins (e.g., IHF) bound on these intermediate regions help to direct DNA looping. Many EBP activators are modular in their structure generally containing three modules or domains: one amino terminal module required for signal sensing, a central domain for ATP hydrolysis and transcription activation, and a carboxy-terminal domain for DNA binding/recognition. As expected, the amino/terminal domain is the least conserved since it adapts to the sensing of diverse signals. Sometimes this amino/terminal domain serves as the receiver module for phosphorylation by the sensor protein in the two-component systems. Either phosphorylation or binding of small molecules induces oligomerization and increases the rate of ATP hydrolysis. This released energy is coupled with the formation of an open-complex promoter possibly through a conformational change in RNAP holoenzyme. The activity of $\sigma^{54}$ promoters requires the activity of EBP. It is notable that EBPs, in some cases most importantly by repression, can function by regulating $\sigma^{70}$ promoters.

\section{The Regulation of Gene Transcription by Nucleoid Structure}

In both archaea and bacteria, the RNAP is present in the cell in limited quantities and most of it is used to channel the synthesis of stable ribosomal RNA, necessary for translation (occupying up to $95 \%$ of the total RNA synthesis in conditions of rapid growth). On the other hand, some RNAP is bound non-productively to the DNA in a nonspecific way. Thus, the availability of RNAP used for transcription of the thousands of genes in a prokaryote cell is limited. In addition, the $\sigma$ factor and its accessory factors are also limited, which generates an intense competition between promoters for the RNAP holoenzyme. These limitations explain why, depending on promoter strength and the TFs availability, it requires a tight regulation and production of many transcripts from some promoters and little or no transcription from others. Here, we describe the main mechanisms that modulate the gene transcription initiation in archaea and bacteria.

\subsection{Transcriptional Regulation by Nucleoid-Structure in Bacteria}

All cells have to replicate a long genome to fit into a small space while maintaining DNA accessibility for replication, transcription, repair and other DNA-related biochemical processes. In eukaryotes the central replication unit of chromatin is the nucleosome, which consists of dimers corresponding to each of the following 
histones: H2A, H2B, H3 and H4, wrapping a DNA segment of around 200 bp, and one segment of $\sim 50 \mathrm{bp}$ DNA is wrapped by H1 histone, linking each nucleosome structure (Nemeth and Langst, 2004; Bernstein and Hake, 2006; Robinson and Rhodes, 2006; Woodcock, 2006). Replication of DNA inside the cell helps maintain the highest level of DNA compactness and controls gene expression by inhibiting access of RNAP to promoters when they are not needed for RNAP expression. In order for transcription to occur, nucleosomes need to be restructured by remodeling enzymes that destabilize these structures and expose the dsDNA. In prokaryotes with genomes ranging from $\sim 0.5$ to $\sim 6 \mathrm{Mbp}$, DNA must be packaged $\sim 1000$ times to constitute the nucleoid that occupies a very small cellular space $(\sim 1 \mu \mathrm{m})$. A bacterial nucleoid contains 50-400 negatively supercoiled DNA loops that are $\geq 10 \mathrm{~kb}$ in size (Carpentier et al., 2005; Hashimoto et al., 2005; Thanbichler et al., 2005; Zimmerman, 2006). These DNA loops might be topologically discrete chromosomal territories independent of each other. The nucleoid is believed to be composed mostly of DNA (60\%), ribosomal complexes and nucleoid associated proteins or TFs. Due to the compactness of a folded genome, it is postulated that gene regulation in eukaryotes and bacteria might face two different scenarios: restrictive and nonrestrictive ground state, respectively (Struhl, 1999). In other words, it is suggested that bacterial DNA is always available for transcription, accessible for competitive binding of TFs and RNAP to operators and promoter regions, whereas eukaryotic DNA is densely packaged into repressive chromatin and therefore is practically inaccessible to both TFs and RNAP. Consistent with this view, it is known that bacterial RNAP has been associated with some $\sigma$ factors that can recognize and trigger transcription by strong promoters, without requiring additional TFs to mediate the process. The eukaryotic RNAPs, however, do not have an intrinsic promoter-binding ability and the initial role of a TF in eukaryotes has been postulated to recruit additional nucleosome-remodeling proteins (e.g., RSC, SWI/SNF) to unwind the DNA and to make the promoters accessible to the RNAP complex. The $\sigma^{54}$ promoters in bacteria behave similar in this sense to one in eukaryotes in that they require TFs to initiate transcription, since RNAP is unable to transcribe even weakly without the presence of a TF. However, in the case of the $\sigma^{70}$, that dominate transcription in bacteria, the TFs are used to strengthen the recognition of weak promoters, whereas in eukarya, the TFs are necessary to recruit the RNAP complex for transcription (Ptashne and Gann, 1997; Ptashne and Gaan, 2002). These different scenarios attributing to the levels of compactness of folded genomes correlate with different mechanisms for transcription regulation. Bacterial genomes can be as much subject to activation as to repression, whereas the role of TFs in eukaryotes is mostly activation.

\subsection{Transcriptional Regulation by Nucleoid-Structure in Archaea}

In the previous section, transcriptional regulation in eukaryotes and bacteria has been reviewed with respect to nucleoid-structure but it is interesting to find out the 
nature of the same mechanism in archaea. RNAP in archaea, even in a simplified way, bears a resemblance to eukarya RNAP whereas the genomic package and TF regulators are more similar to those of bacteria. All the species of archaea studied up to date contain abundant quantities of small DNA-binding proteins ( $\mathrm{SuI} 7 \mathrm{~d}$, Alba, histones, HTa, MC1), whose binding seems to be sequence-independent, and in both chrenarchaea and euryarchaea these have been localized in vivo in the nucleoid (Herrmann and Soppa, 2002; Long and Faguy, 2004; Majernik et al., 2005). Nonetheless, there is no evidence for a universal mechanism for archaeal chromatin structure (Hayat and Mancarella, 1995; Poplawski and Bernander, 1997; Hoppert and Mayer, 1999; Malandrin et al., 1999; Bernander, 2000; Majernik et al., 2005). For euryarchaeal genomes, it has been suggested that the histone, a small protein, is conserved only in its folding domain but not the accessory domains, for acetylation or methylation, like their eukaryal counterparts (Reddy and Suryanarayana, 1989; Grayling et al., 1996). In addition, bioinformatics analyses indicate that the DNAsequence that facilitates DNA-binding and wrapping by histones is statistically over-represented in this archaeal genome but there is no definitive evidence for the constitution of chromatin structures. Furthermore, there are no conclusive evidences regarding whether or not these chromosomal regions (for predicting histone-binding in euryarchaeal genome) are related to zones of transcription. Thus, transcription regulation in archaea seems to be more related to bacteria mostly depending on TF activity following the nonrestrictive model. However, the role of these small DNA-binding proteins in the transcriptional regulation is still not clear.

\subsection{Nucleoid-Associated Proteins in Bacteria}

In bacteria, the so-called Nucleoid- Associated Proteins (NAPs) contribute to the replication of DNA as well as to transcriptional regulation, including the DNAbridging protein (H-NS) and the DNA-bending proteins (IHF, HU and FIS), (Kepes, 2004; Dame, 2005; Luijsterburg et al., 2006).

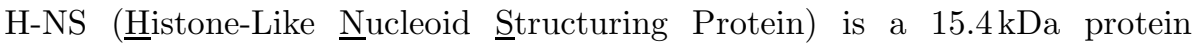
conserved among Gram-negative bacteria. The active form of H-NS is believed to be a dimer or larger oligomers. This protein binds DNA without clear sequence specificity but with some bias to curved DNA or to more flexible than normal DNA. The binding of this protein to DNA results in bridges between adjacent DNA duplexes that provide the structural basis for transcriptional repression (Navarre et al., 2006). In exponentially growing cells (when it is maximally expressed in $E$. coli), this protein reaches the concentration of one dimer per $1400 \mathrm{bp}$ of DNA.

IHF (Integration $\underline{H}$ ost Factor) functions as an accessory factor in a wide variety of processes such as replication, site-specific recombination and transcription. It is a heterodimer of two subunits (with $25 \%$ sequence homology between them): $\alpha$ subunit of $11 \mathrm{kDa}$ and $\beta$ subunit of $9 \mathrm{kDa}$ in size. This heterodimeric protein binds to specific DNA sequences with nanomolar affinity. Expression of IHF is maximal during stationary growth (1 IHF heterodimer/335 bp of DNA), and is predicted to 
have $\sim 1000$ binding sites on the $E$. coli genome. IHF binding occurs frequently upstream of the $\sigma^{54}$ promoters in Klebsiella and other bacteria, and is essential for the bending required to bring the remotely bound TF or enhancer-like regulator physically close to the polymerase bound at the $\sigma^{54}$ promoter. That is, it provides a mechanism of specificity to diminish cross-talk or undesired activation of other $\sigma^{54}$ promoters nearby (Gralla and Collado-Vides, 1996).

The Histone-like protein from E. coli strain $\underline{\mathrm{U}} 93(\mathrm{HU})$ is present in most of bacteria as $\alpha$ and $\beta$ homodimers (each $9.5 \mathrm{kDa}$, with $70 \%$ homology between them). HU binds non-specifically to DNA, but has a higher affinity for the supercoiled and distorted DNA. The DNA-bending induced by HU is suggested to be less rigid than that induced by IHF and is considered as a flexible hinge. Interestingly, HU activity can be substituted by the eukaryotic ㅂigh 쓰obility Group Box 1 protein (HMGB1), an extranuclear cytokine with chromosome remodeling properties (Perez-Martin and De Lorenzo, 1997). HU protein reaches its maximal production during the exponential phase $(1 \mathrm{HU} / 550 \mathrm{bp})$.

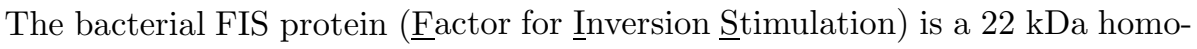
dimeric complex that binds DNA in a sequence-specific manner. Strong binding sites for FIS are located upstream of a number of stable RNA operons where it acts as a transcriptional activator. FIS is the most abundant NAP at the beginning of exponential growth (1 FIS/450 bp) and is completely absent during the stationary phase of growth.

Some nucleoid-associated proteins are expressed during all phases of bacterial growth (Grainger et al., 2006; Luijsterburg et al., 2006). All these proteins favor DNA packing and can, at the same time, affect transcriptional regulation. In contrast to eukaryotic genomes, the DNA-binding sites for the bacterial NAPs are biased toward non-coding parts of the genome. These proteins, also recognized as regulators of transcription, are not modulated by allosterism through the direct binding/sensing of environmental or metabolic signals as the majority of the classic TFs. It is, however, postulated that these NAPs sense the state of the chromosome and act as global regulators by permitting or restricting the access to binding sites of RNAP or additional TFs.

It is interesting to note that, in this context, a large number of CRP sites have been found by ChIP-chip experiments (Grainger et al., 2005). Because of their genomic positions, only a fraction of them corresponds to the CRP as a transcriptional regulator, whereas other fractions within the coding region may have different roles. The large number of CRP sites suggests that these and other global regulators multimerize on contiguous DNA binding sites and could function by specifically modifying some chromosomal regions depending on the presence and absence of environmental signals (as cAMP for CRP).

Generalizing this idea a step further, it is certainly interesting to note that most of the proposed global regulators of E. coli (Martinez-Antonio and Collado-Vides, 2003) multimerize and show some DNA-structuring properties by binding the DNA similarly to the nucleoid-associated proteins. Earlier, all TFs had been considered as 
transcription regulators, but the function of CRP has opened new avenues or, more precisely, introduced an additional means to globally affect transcription indirectly other than its direct role in modulating the transcription initiation.

Furthermore, transcription of NAPs is regulated by other TFs sensing environmental or endogenous signals, which led to the conclusion that the activity of NAP is indirectly coupled to the cellular or milieu conditions. In this way, one can envision alternative states or configurations of the bacterial chromosomal structure, that, depending on the growing conditions, determine the global pattern of gene transcription; e.g., exponential versus stationary phase (Travers and Muskhelishvili, 2005).

\section{Post-transcriptional Control Mechanisms for Regulating Gene Expression}

\subsection{Riboswitches}

Riboswitches (RNA-switches) are found in the $5^{\prime}$ un-translated leader regions (UTR) present in some mRNA molecules. They directly bind to a small target molecule which can affect a gene's expression. Thus, mRNA that contains a riboswitch is directly involved in regulating its own activity, depending on the presence or absence of its target molecule (Winkler and Breaker, 2005; Batey, 2006; Nudler, 2006). This small-molecule sensing region on mRNA is named aptamer, and it undergoes structural changes when bound to their specific small molecule. These structural changes affect the expression of a gene in the following ways:

1. by the formation of transcription termination hairpins;

2. by sequestering the ribosome-binding site (Shine-Dalgarno), thereby blocking translation; and

3. by self-cleavaging (i.e. the riboswitch contains a ribozyme that cleaves itself in the presence of sufficient concentrations of its metabolite).

The small metabolites sensed by these mRNA structures include vitamins, cofactors, amino acids, sugars and nucleotides. Their presence has been experimentally proven in bacteria and in some cases in archaea as well as eukarya.

\subsection{Attenuators}

An attenuator is an RNA sequence that regulates the expression of certain genes by terminating transcription. A transcriptional attenuator usually depends on the formation of mutually exclusive secondary structures (Matsumoto et al., 1986; Switzer et al., 1999). It requires a "rho-independent" (or intrinsic) terminator, which, when formed, causes the RNA polymerase to prematurely stop transcribing. An anti-terminator is a structure that impedes the formation of the terminator, and 
an anti-anti-terminator is a structure that impedes the formation of the antiterminator. In this fashion, the most stable structure in a given condition will form and govern the outcome of the expression of the gene(s) immediately downstream. Different structures may be formed under different conditions such as when a protein, the ribosome or a small molecule stabilizes one of the structures.

\subsection{Non-coding $R N A s$}

A non-coding RNA (ncRNA) is any RNA molecule that is not translated into a protein (see Chapter 2 for more details). ncRNAs have been found to have roles in a great variety of biological processes, including transcriptional regulation, chromosome replication, RNA processing and modification, messenger RNA stability and translation, and even protein degradation and translocation (Gottesman, 2005; Vogel and Papenfort, 2006). Recent studies indicate that ncRNAs are far more abundant and important than initially imagined. Some of the known mechanisms for ncRNAs activity include the following:

(i) Directing base-pairing with target RNA or DNA molecules is central to the function of some ncRNAs in repressing translation by forming base pairs with the Shine-Dalgarno sequence and occluding ribosome binding.

(ii) Some ncRNAs mimic the structure of other nucleic acids as bacterial RNA polymerase may recognize the $6 S$ RNA as an open promoter, and bacterial ribosomes recognize tRNA as both a tRNA and an mRNA.

(iii) Some ncRNAs function as an integral part of a larger RNA-protein complex, such as the signal recognition particle whose structure has been partially determined (Gottesman, 2005; Winkler, 2005; Vogel and Papenfort, 2006). In addition, the conserved RNA-binding protein, Hfq, functions as a pleiotropic regulator that modulates the stabilization or translation of an increasing number of mRNAs (Valentin-Hansen et al., 2004; McNealy et al., 2005; Sittka et al., 2006).

Regulon DB, the database on transcriptional regulation in E. coli, now contains the set of small RNAs and has been curated to include information on their corresponding target sites and regulated genes.

\section{4. mRNA Half-Life}

The life of an mRNA molecule in prokaryotes begins with transcription and ultimately ends in degradation. In E. coli, around $80 \%$ of mRNAs have half-lives between 3 and $8 \mathrm{~min}$ (Bernstein et al., 2002; Selinger et al., 2003). The stability of mRNA in prokaryotes depends on multiple cis-elements and trans-factors. The trans-acting proteins are mainly nucleases and the cis-acting elements that protect 
mRNA from degradation are stable stem-loops at the $5^{\prime}$ end. However, it seems that transcription is the dominant factor in determining the mRNA steady-state level in $E$. coli and that the variation in their half-life may have an alternative biological role, perhaps to facilitate transient changes in mRNA abundance in response to specific environmental perturbations or the progress of bacteria through the celldivision cycle. We have now a hint for archaea, with the global measures of half-life mRNA in Sulfolobus and Halobacterium archaea; their average mRNA half-life is 5 and $10 \mathrm{~min}$, respectively, similar to their bacterial counterparts (Andersson et al., 2006; Hundt et al., 2007).

\section{Regulatory Network Integration}

\subsection{Global and Local TFs}

The nature of biological networks presents a scale-free distribution with a few elements highly connected while most of them sparsely connected within the network (Barabasi and Albert, 1999; Hartwell et al., 1999). In the transcriptional network, this distribution is marked by the presence of a few TFs defined as global TFs presenting the following properties: (i) they have few paralogs; (ii) they regulate many genes; (iii) they regulate several genes encoding for other TFs; (iv) they cooperate with numerous TFs and together regulate other genes; (v) they directly affect the expression of a variety of promoters that use different $\sigma$ factors; and (vi) they regulate genes from different functional classes (Martinez-Antonio and Collado-Vides, 2003). On the other hand, local TFs mostly respond to defined environmental signals, constituting small regulons, and normally fall to the lowest level in the transcriptional TFs hierarchy. Global TFs regulate and co-regulate with local TFs coordinating the cellular transcriptional response with general and specific transcriptional conditions (Covert et al., 2004; Barrett et al., 2005; MartinezAntonio et al., 2006; Yu and Gerstein, 2006; Janga et al., 2007b).

\subsection{Noisy Gene Transcription}

The availability of new methodologies has made possible to follow gene expression patterns even at the single-cell level (Swain et al., 2002; Becskei et al., 2005; Golding et al., 2005; Hooshangi et al., 2005; Rosenfeld et al., 2005; Cai et al., 2006). Previous studies have shown that gene expression occurs as pulses or bursts of transcription, and that the level of response of a gene is highly variable when comparing individual cells in a population. A heterogeneous population exhibits heterogeneous transcription mechanisms in a cell population. As a consequence, in a population, there are more than one cellular configuration even in the same growing conditions identified as phenotypes. These numerous phenotypes contribute to the prokaryotes plasticity required for environmental adaptation and population robustness. 


\subsection{Combinatorial Regulation of TFs}

Though regulation by multiple TFs is more frequent in eukaryotes than in prokaryotes, this is also the dominant form of gene regulation in prokaryotes where multiple TFs work in collaboration (Adhya, 2003; Martinez-Antonio and ColladoVides, 2003). In this way, multiple signaling pathways and cellular conditions can converge on the regulation of transcription units whose protein products can respond to these conditions. For example, the $\operatorname{sod} A$ operon of $E$. coli is regulated by 6 different TFs sensing internal and external signals. In most of genes, transcription co-regulation is coupled with the activity of internal and external sensing TFs or with nucleoid-associated proteins.

The following groups of genes have been defined based on their co-regulation and co-expression.

\subsubsection{Regulon}

A regulon is the set of genes subject to regulation by only one regulator (Maas and McFall, 1964). The initial definition was derived after studies of the arginine biosynthetic genes, which were found to be scattered (or non-contiguously located) in the chromosome of $E$. coli in contrast to operons. To better describe this type of group of co-regulated genes, we call this a simple regulon (as opposed to a complex regulon). A complex regulon is a group of genes subject to regulation by two or more regulators, where all genes are subject to regulation by exactly the same TFs. A strict complex regulon otherwise is a set of genes where the effect of each regulator (activator or repressor) is the same for all the regulated genes (GutierrezRios et al., 2003). For more detailed discussion on regulons, we refer the reader to Chapter 11.

\subsubsection{Sigmulon and Stimulon}

A sigmulon is a term used to refer to the group of genes transcribed by a common $\sigma$ factor (i.e. $\sigma^{70}, \sigma^{54}$, etc.). It implies that the encoding TUs have common promoters to be recognized by the same $\sigma$ factor but not necessarily to be transcribed at the same time. A related term is stimulon that refers to the collection of genes (or operons, regulons) undergoing regulation by the same stimulus and is generally used for prokaryotic systems, for example quorum sensing, heat shock, etc. (Cases et al., 2003b; Schumann, 2003).

\subsection{Transcriptional Regulatory Network}

A typical prokaryotic cell might be regarded as a small bag of thousands of cellular components each devoted to different tasks but interacting with each other (Hartwell et al., 1999; Barabasi and Oltvai, 2004). The cellular interactions are commonly known as a network where the nodes represent the cellular components and the edges their functional interactions. Focusing on transcriptional regulation, 
we can draw the transcribed products of TUs as the nodes and the regulatory activities of TFs on the transcription of those genes as the edges. In this manner we can represent the regulatory interactions between TFs and their target genes; because some of those genes self-regulate (auto-regulation) or regulate other TFs, we can have a hierarchical transcriptional regulatory network (Martinez-Antonio and Collado-Vides, 2003; Resendis-Antonio et al., 2005; Yu and Gerstein, 2006). The availability of many individually described regulatory interactions for TUs and highthroughput experimental data analysis (micro-arrays and ChIP-chip experiments) mainly in $E$. coli and other organisms provided a wealth of data that permit analyses of organisms from a biological point of view; an approach that combines mathematical modeling and prediction of molecular interactions to be verified experimentally in an integrated way. In these reconstructed regulatory networks, it is possible to define which TFs work together, jointly defining regulatory modules because they constitute groups of TFs processing similar signals, and generate coordinated transcriptional responses; their biological redundancy many contribute to the system's robustness (Thieffry and Romero, 1999; Ihmels et al., 2002; Segal et al., 2003).

It is also possible to define small topological units in a network that are overrepresented relative to a randomly constituted network, and that contain at least three or four genetic elements named network motifs (Milo et al., 2002; Shen-Orr et al., 2002). Understanding how these topological structures evolve and function in the genetic networks helps in the better understanding of biological systems. In addition to genetic components in the network we can add the effector metabolites, signaling cascades, epigenetic regulations, etc., which make the network analysis complicated but are indispensable to achieve a better comprehension of the functions of biological systems.

\section{Summary}

In this chapter, we have summarized the diversity of mechanisms with a range of combinatorial components that increase the flexibility both of what constitutes a specific promoter, for instance in the $\sigma^{70}$ architecture, as well as of what constitutes a particular regulatory region, with its combined set of regulating TFs. These combinations provide room for highly selected specific systems in terms of their integration with the system and the kinetics of their responses. The work of evolution also provides a unique rationale for understanding gene regulation, be it in bacterial, archaeal or eukaryotic cells, where regulation serves as a molecular switch executed by specific molecules that provide alternative conformations -active and inactive- which are themselves connected through "sensing" pathways to the internal and or external changing conditions.

We have also described how genes are organized for transcription in prokaryotic organisms and the main machinery for processing cis-regulatory signals and transregulatory factors is needed for the proper regulation of genes in response to new or changing environmental conditions. In archaea, the basal machinery for 
transcription is similar to the RNAP II of eukarya, while the use of TFs for modulating the gene transcription is similar to bacteria. Obviously, our knowledge about transcriptional regulation in archaea is rather limited as compared to bacteria due to the difficulty to grow them in the laboratory. Both archaea and bacteria represent simple successful cellular systems adaptable to many environmental conditions and understanding their functions is fundamental to the understanding of our changing environment throughout the history of life on the earth.

\section{Further Reading}

Browning, DF, Busby, SJ (2004) The regulation of bacterial transcription initiation. Nat Rev Microbiol 2(1):57-65.

Collado-Vides, J, Magasanik B, Gralla JD (1991) Control site location and transcriptional regulation in Escherichia coli. Microbiol Rev 55(3):371-394.

Gruber TM, Gross CA (2003) Multiple sigma subunits and the partitioning of bacterial transcription space. Annu Rev Microbiol 57:441-466.

Ptashne M, Gann A (1997) Transcriptional activation by recruitment. Nature 386(6625):569-577.

Ptashne M, Gaan A (2002) Genes and Signals: Cold Spring Harbor Laboratory Press.

Struhl K (1999) Fundamentally different logic of gene regulation in eukaryotes and prokaryotes. Cell 98(1):1-4.

Luijsterburg MS, Noom MC, Wuite GJ, Dame RT (2006) The architectural role of nucleoid-associated proteins in the organization of bacterial chromatin: a molecular perspective. J Struct Biol 156(2):262-272.

\section{Acknowledgments}

The authors acknowledge two anonymous referees as well as the editors, whose suggestions and comments helped to improve this chapter. J.C.-V. acknowledges NIH grant R01 GM71962-03. 